\title{
PESQUISA ONLINE DE USABILIDADE: PROCESSO DE COMPRA NOS WEBSITES COMPRA FÁCIL E PONTO FRIO POR USUÁRIOS ACIMA DE 52 ANOS
}

Online Usability research: buying process on websites Easy Purchase and Ponto Frio by users above 52 years

\section{FERNANDES, Fabiane Rodrigues I Doutoranda em Design}

Faculdade de Administração e Artes de Limeira

fabyfernandes@gmail.com

\section{Resumo}

Este artigo apresenta uma pesquisa online de usabilidade do processo de compra em dois websites: Compra Fácil e Ponto Frio. A usabilidade é medida através de escalas de pontuação e das métricas de eficácia, eficiência e satisfação (NBR 9241-11), por meio de uma avaliação empírica. Os resultados demostram que ambos websites não conseguem atingir uma margem considerada aceitável, segundo modelo de BANGOR; KORTUM; MILLER, 2009.

Palavras-chaves: Avaliação empírica. Usabilidade. Interação. Websites. Compras.

\section{Abstract}

This paper presents an online survey of usability of the buying process in two websites: Compra Fácil and Ponto Frio. Usability is measured by scoring scales and effectiveness metrics, efficiency and satisfaction (NBR 9241-11) by means of an empirical evaluation. The results demonstrate that both sites are not able to reach a margin considered acceptable, according to model BANGOR; KORTUM; MILLER, 2009.

Keywords: Empirical evaluation. Usability. Interaction. Websites. Shopping. 


\section{INTRODUC̣̃̃O}

Nas últimas décadas a população brasileira praticamente dobrou em relação aos 90 milhões da década de 1970, segundo dados obtidos pelo Instituto Brasileiro de Geografia e Estatística (IBGE, 2004). Existe a previsão de que, em 2050, serão 259,8 milhões de brasileiros, cuja expectativa de vida ao nascer será de 81,3 anos, a mesma dos japoneses hoje. Em 2000, o grupo de 0 a 14 anos representava $30 \%$ da população brasileira, enquanto que, os maiores de 65 anos eram apenas 5\% da população. Acredita-se que, em 2050, esses dois grupos etários se igualarão e cada um deles representará $18 \%$ da população brasileira. Segundo o Censo (IBGE, 2002), a proporção de pessoas da terceira idade vem crescendo mais rapidamente que a proporção de crianças, sendo a principal responsável pela queda da taxa de fecundidade, enquanto que, a longevidade vem contribuindo progressivamente para o aumento de pessoas da terceira idade na população.

Com a evolução digital e o crescente número de informação disponibilizada no ambiente virtual, podemos afirmar que, nos dias de hoje, as pessoas da terceira idade se comportam de forma mais sociável que as de outras gerações, antecessoras ao surgimento da Web. Guasti (2011), diretor geral da e-bit, afirma que, segundo estudo levantado pela empresa, 9\% dos e-consumidores que afirmaram terem sido influenciados por alguma rede social, no momento da compra, tinham entre 50 e 64 anos. Isso demonstra uma crescente tendência de pessoas "mais velhas" conectadas às mídias sociais.

Em relação a compras virtuais, os consumidores mais maduros têm uma forte participação comprovada por números. Segundo dados da E-bit ${ }^{30}$, atualmente $25 \%$ dos e-consumidores informam ter mais de 50 anos. Em 2001, a terceira idade era $10 \%$ da população de e-consumidores, o que pode transmitir uma maior confiança desse público em fazer suas compras pela Internet, além de não precisarem se locomover ou passar por situações cansativas ou de stress ao saírem de casa para comprarem seus produtos. Esta participação deve continuar crescendo na próxima década, acompanhando a mudança da pirâmide etária brasileira.

\section{O idoso e a internet}

As gerações mais velhas (acima dos 54 anos) ${ }^{31}$ estão dispostas, receptivas e são capazes de aprender coisas novas, porém, estão mais susceptíveis a ansiedade e diminuição da confiança em suas habilidades para usar as 30 E-bit é um website que avalia os websites de compras online, oferecendo um selo de classificação. anos) e G.I. Generation (acima de 73 anos). (PEW INTERNET, 2009).

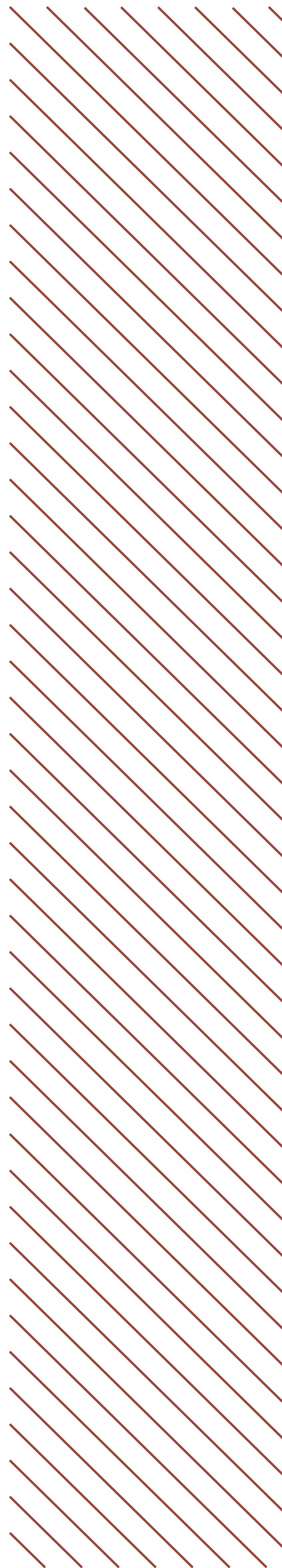


tecnologias com sucesso (CZAJA, 2005). Zajicek (2004) afirma que designers de produtos interativos devem levar em conta as necessidades especiais de uma parte da população que, muitas vezes, sentem dificuldades de usar produtos atuais. Este mesmo autor alerta que não fazer isso resultará em um grupo grande e crescente de cidadãos marginalizados e excluídos do mundo cotidiano atual. É preciso lhes permitir continuar a viver de forma independente (ZAJICEK, 2004).

Para Phiriyapokanon (2011) quando nos referimos às pessoas idosas, estamos nos referindo ao grupo mais velho e usuários mais antigos. Por outro lado, os usuários mais velhos podem ser relacionados às pessoas com declínio da capacidade relativo à idade. No trabalho de Hawthorn (2006), o mesmo menciona que efeito do envelhecimento começa a se tornar perceptível por volta dos 40 anos de idade. Na grande parte da literatura o grupo mais velho é formado por pessoas depois de 60 anos idade. Porém vários estudos relacionados ao uso de computadores e Internet por usuários mais velhos utilizam pessoas com mais de 50 anos como participantes dos estudos (NAUMANEN; TUKIAINEN, 2007; LUNN; HARPER, 2011).

\section{O design e a usabilidade}

Paschoarelli e Silva (2006) caracterizam o Design Ergonômico como sendo o processo de design, cujo princípio é a aplicação da ergonomia no desenvolvimento de artefatos tecnológicos, com o objetivo de obter produtos e sistemas mais seguros, confortáveis, eficientes e aceitáveis. O Design Ergonômico, então, se baseia na inter-relação do design, ergonomia e usabilidade. $E$, porque não dizer, que também faz parte desta relação à experiência do usuário.

Para se garantir um bom Design é indispensável que Design, Ergonomia, Experiência do Usuário e Usabilidade sejam parte de um mesmo processo, que visa garantir um resultado que satisfaça as pessoas. Se, pensarmos, que um bom Design é o processo de resolução de um problema ou necessidade através de um artefato, que atenda de forma satisfatória o usuário, então, é necessário que o processo de Design faça uso de critérios de Ergonomia, que visa adaptar o ambiente construído as necessidades e anseios dos usuários, para obter o resultado satisfatório. E, para se adaptar aos usuários é necessário compreender a experiência do usuário, ou seja, como é a relação de uso e como se dar o processo de uso do ambiente construído e seus artefatos, as expectativas, a qualidade de uso (usabilidade) e as impressões (satisfação), resultantes da experiência vivenciada com essa interação. O Design, então, fará uso na configuração estético-funcional do artefato, de critérios ergonômicos e dados da experiência do usuário, para entregar uma solução satisfatória 
(FERNANDES, 2013).

\section{AVALIAÇÃO EMPÍRICA}

Baseado na definição de Usabilidade da International Standards Organization (ISO 9241-11), que é a medida de uso de um produto por usuários específicos que buscam atingir objetivos específicos com eficácia, eficiência e satisfação em um contexto específico de uso, é possível realizar uma avaliação global de uma interface. Dentre as formas de avaliação da Usabilidade, a ISO 9241 (ASSOCIAÇÃO BRASILEIRA DE NORMAS TÉCNICAS, 2002) pode ser utilizada em projetos, como uma ferramenta para avaliação da Usabilidade. Essa norma possui vários tópicos que tratam de aspectos ergonômicos do uso de softwares e hardwares. Em seu tópico 11, a norma trata da medida de Usabilidade global, através das medidas de eficácia (objetivos alcançados com sucesso), eficiência (tarefas completadas por unidade de tempo e outras variáveis) e satisfação (através de um questionário).

Existem vários métodos de avaliação da usabilidade que podem se enquadrar em dois tipos: métodos de inspeção, que são avaliações feitas por especialistas, como o caso da avaliação heurística (NIELSEN; MOLICH, 1990), através de critérios e guidelines ou métodos empíricos, que consiste em uma avaliação, no qual o usuário faz uso do sistema/produto, realizando algumas tarefas pré-determinadas. Normalmente, as avaliações usando o método empírico são acompanhadas pelo avaliador e gravadas. Mas, além das avaliações em laboratórios de usabilidade é possível realizar uma avaliação de usabilidade através de uma pesquisa online. Albert, Tullis e Tedesco (2013) acreditam que felizmente, nos últimos anos, testes on-line de usabilidade surgiram para atender necessidades críticas como tempo e custos, sendo capaz de obter informações valiosas sobre a experiência do usuário.

\section{O número de participantes}

Alguns pesquisadores da área de Usabilidade, como Nielsen e Landauer (1993) acreditam que, com cinco participantes em uma pesquisa já é possível identificar $80 \%$ dos problemas de uma interface, relacionados à qualidade de uso, por isso é conhecido como "o número mágico" (TULLIS; ALBERT, 2008). Alguns pesquisadores mais recentes já não acreditam que cinco participantes seja uma quantidade satisfatória, porém, Tullis e Albert (2008) acreditam que esta quantidade pode ser satisfatória, pois, na maioria dos testes de usabilidade realizados por eles, independentemente do número total de participantes, a maioria dos problemas mais significativos de usabilidade se mostram a partir do quarto ou quinto participante. Assim, esses mesmos autores acreditam que o "número mágico" de participantes serve quando o escopo da avaliação é limitado (conjunto limitado de funções) e quando o público-alvo de usuários é bem definido e representativo. 


\section{MATERIAIS E MÉTODOS}

Essa pesquisa faz parte de uma pesquisa maior sobre a avaliação do uso de websites de compras online por usuários "mais velhos". Foi utilizado como técnica de pesquisa questionários, elaborados pela pesquisadora por meio de um modelo de protocolo, tendo como fonte de informação a pesquisa bibliográfica.

Os procedimentos foram baseados em recomendações éticas do Conselho Nacional de Saúde (BRASIL, 1996) e os parâmetros éticos foram baseados na Norma "ERG BR 1002 - Código de Deontologia do Ergonomista Certificado" (ASSOCIAÇÃO BRASILEIRA DE ERGONOMIA, 2003). Todos os sujeitos leram e assinaram o Termo de Consentimento Livre e Esclarecido (TCLE). Para a presente pesquisa foi utilizado computadores com acesso a Internet e protocolos de coleta de dados, separados da seguinte forma:

- TCLE - Termo de Consentimento Livre e Esclarecido: protocolo que tem como objetivo informar sobre a pesquisa e que a mesma não causará nenhum dando ao participante e outras informações que podem ser consideradas como importante;

- Protocolo de Identificação e Experiência da Internet: protocolo que busca compreender de cada participante, sua idade, formação e conhecimentos sobre a Internet;

- Protocolo de Ensaio de Interação com o website Compra Fácil: protocolo de coleta de informação sobre a interação com o website compra fácil;

- Protocolo de Ensaio de Interação com o website Ponto Frio: protocolo de coleta de informação sobre a interação com o website ponto frio.

\section{Os sujeitos}

Participaram da pesquisa de usabilidade, realizada através de um protocolo disponibilizado online, no mês de janeiro de 2012, cinco voluntários com idade média de 60 anos (d.p. 3,74), sendo 02 do gênero masculino e 03 do gênero feminino. Quinze protocolos não foram entregues ou finalizados. Dois protocolos foram descartados por não atingirem a idade mínima para participação.

\section{A coleta de dados}

A coleta de dados inicia-se com o protocolo respondido pelos sujeitos da pesquisa. Todos os protocolos on-line preenchidos pelos participantes da pesquisa chegavam à caixa de e e-mail da pesquisadora constando data e horário do preenchimento e o número de IP (Internet Protocol) que é o número 
de identificação da rede ou computador de onde foi enviado o protocolo. Primeiramente chegava ao e-mail o TLCE preenchido, seguido tempo depois do protocolo de interação com os dois websites de compra online (Compra Fácil e Ponto Frio) preenchido.

A pesquisa que busca compreender como ocorre a relação de uso dos websites de compra online por usuários acima de 52 anos, baseou-se em um ensaio de interação que consistia na realização de uma tarefa em ambos os websites, que seria a compra de três produtos dentro de uma faixa de preço.

\section{A tarefa}

- A tarefa de realizar uma compra nos websites pesquisados nessa dissertação consistia de 07 subtarefas:

- Comprar uma pendrive com preço até $\mathrm{R} \$ 80,00$;

- Comprar um umidificador de ar até $\mathrm{R} \$ 220,00$;

- Comprar uma impressora multifuncional até $\mathrm{R} \$ 450,00$.

- Finalizar o pedido;

- Inserir dados de cadastro, conforme informado no protocolo de pesquisa. Um cadastro já havia sido realizado pelo laboratório para que os voluntários não precisassem inserir seus dados e momento algum;

- Escolher forma de pagamento "boleto bancário". Para que os usuários não precisassem informar seus dados ou realizar a compra verdadeiramente, optou-se pelo boleto, pois o mesmo é emitido sem dados do comprador e expira em três dias se não for pago. Foram contatadas as centrais de atendimento de ambos os websites pesquisados informando que o cadastro criado seria usado em uma pesquisa e que tanto os produtos comprados quanto os boletos gerados não seriam compras verdadeiras;

- Visualizar confirmação de pedido.

\section{Análise dos dados}

O estudo da avaliação empírica baseou-se na inferência estatística (TULLIS; ALBERT, 2008). Com a intensão de definir parâmetros para a discussão será utilizado como referência para os resultados uma escala de pontuação definida por Bangor, Kortum e Miller (2009). Uma escala padrão de pontuação muito aceitável (BANGOR; KORTUM; MILLER, 2009) é que os produtos que obtiveram pontuação superior a $90 \%$ podem ser considerados excepcionais, aqueles que obtiveram pontuação igual a $80 \%$ e inferior a $90 \%$ são considerados bons, e os produtos que marcarem uma pontuação entre $70 \%$ - 79\% são aceitáveis. Qualquer pontuação abaixo de 70\% significa problemas de usabilidade e devem ser vistos com preocupação.

Os dados finais serão calculados em porcentagem com base na escala de pontuação do SUS (TULLIS; ALBERT, 2008), conforme método já aplicado em 
uma pesquisa de experiência do usuário por Fernandes e Paschoarelli (2013).

Após preencher o protocolo de identificação o participante era convidado a participar de um ensaio de interação, cuja tarefa já foi descrita. Sequencialmente após realizar a ação pedida no protocolo, o usuário ia preenchendo as informações sobre essa interação. Ao final da interação o usuário informava o número que aparecia na tela de visualização da confirmação do pedido.

\section{Eficácia}

Para cálculo da eficácia foi considerado a tarefa concluída com sucesso, que se baseia na conclusão da compra dos três produtos e confirmação através do número do pedido anotado no protocolo pelo participante. Para essa análise será usada uma escala de dois pontos, sendo que a pontuação 0 (zero) equivale a "não concluída corretamente" e a pontuação 1 (um) equivale a "concluída corretamente". A tabela 1 apresenta o método de pontuação para análise da eficácia para a tarefa realizada em ambos websites. A pontuação final máxima de 6 (seis) pontos ( $p_{\text {máximo }}$ ) equivale a $100 \%$ de eficácia da tarefa. O resultado final é apresentado em porcentagem, através de uma fórmula inserida no software Excel $^{\circledR}$ (=média [soma dos pontos de cada participante] ${ }^{\star} 16,666666667 / 100$ ). Que calcula a média dos pontos em porcentagem ( Média $_{\text {porcentagem }}=$ Média $_{\text {pontos }}$

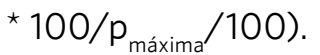

Tabela 1 - Quadro de pontuação para o cálculo da eficácia

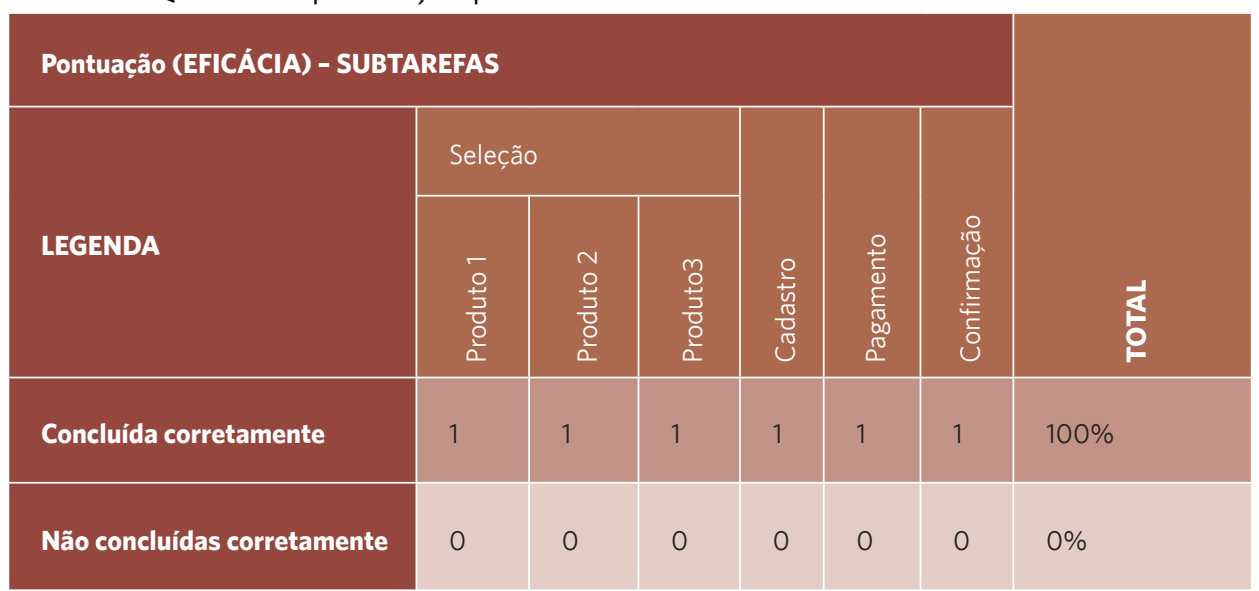

Fonte: Do autor.

\section{Eficiência}

Para o cálculo da eficiência foi considerado as tarefas concluídas com sucesso acrescidas das variáveis tempo e grau de dificuldade. O protocolo de interação abordou uma escala de nove pontos (do 0 ao 8) a ser considerada para análise de cada subtarefa, como mostra a tabela 2 , sendo que a pontuação O (zero) equivale a subtarefa "não concluída corretamente", a pontuação 1 (um) equivale a subtarefa concluída "com dificuldade e com mais de 15 minutos", 
a pontuação 2 (dois) equivale a subtarefa concluída "com dificuldade e entre 05-10 minutos", a pontuação 3 (três) equivale a subtarefa concluída "com dificuldade e entre 02-04 minutos", a pontuação 4 (quatro) equivale a subtarefa concluída "com dificuldade e até 01 minuto", a pontuação 5 (cinco) equivale a subtarefa concluída "com facilidade e com mais de 15 minutos", a pontuação 6 (seis) equivale a subtarefa concluída "com facilidade e entre 05-10 minutos", a pontuação 7 (sete) equivale a subtarefa concluída "com facilidade e entre 02-04 minutos" e a pontuação 8 (oito) equivale a subtarefa concluída "com facilidade e até 01 minuto". Todas as subtarefas finalizadas com dificuldade ficam com pontuação mais baixa que as subtarefas finalizadas com facilidade por se tratar de uma percepção do usuário no momento da interação, já que o tempo perdido pode ter diversas causas como baixa conectividade ou sinal da rede, entre outros. A pontuação final máxima de 48 (quarenta e oito) pontos (pmáxima) equivale a 100\% de eficiência da tarefa, ou seja, as seis subtarefas receberam então pontuação máxima igual a oito pontos. O resultado final é apresentado em porcentagem, através de uma fórmula inserida no software Excel $^{\circledR}$ (=média [soma dos pontos de cada participante] ${ }^{\star} 2,083333333 / 100$ ). Que calcula a média dos pontos em porcentagem (Média ${ }_{\text {porcentagem }}=$ Média $_{\text {pontos }}{ }^{*}$ 100/p máxima $/ 100)$.

\section{Tabela 2 - Quadro de pontuação para o cálculo da eficiência}

\begin{tabular}{|c|c|c|c|c|c|c|c|c|c|}
\hline \multicolumn{10}{|c|}{ Pontuação (EFICÁCIA) para cada subtarefa } \\
\hline 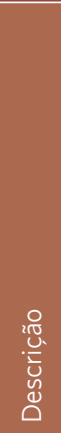 & 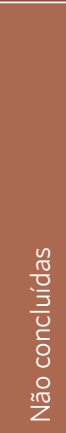 & 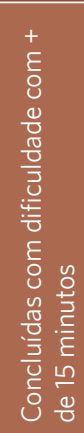 & 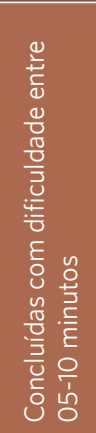 & 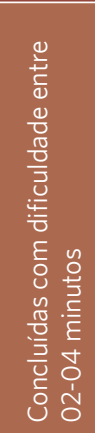 & 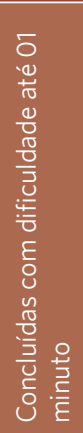 & 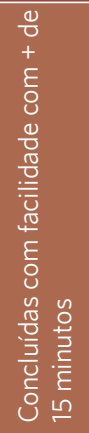 & 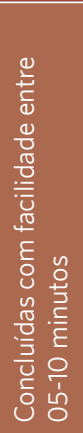 & 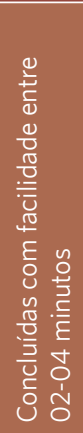 & 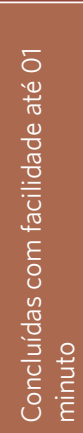 \\
\hline 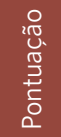 & 0 & 1 & 2 & 3 & 4 & 5 & 6 & 7 & 8 \\
\hline
\end{tabular}

Fonte: Do autor.

\section{Satisfação}

Para o cálculo da satisfação um questionário contendo 14 questões foi aplicado, sendo que, na primeira parte do questionário as seguintes pontuações são utilizadas: a questão 01 trabalha uma escala de dois pontos, sendo 0 (zero) para "ineficiente" e 1 (um) para "eficiente". A questão 02 não tem pontuação, pois apenas quer saber se o participante já conhecia os dois websites. As questões

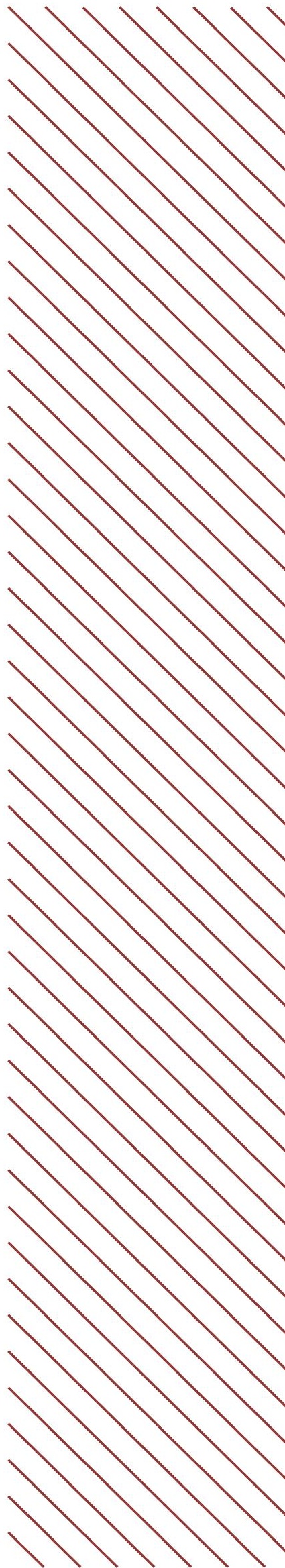


03-04 utilizam, também, uma escala de dois pontos, sendo 0 (zero) para "não" e 1 (um) para "sim". Na segunda parte do questionário foi aplicado o SUS [24]: dez afirmativas alternadas entre positivas e negativas, com escala de Likert ${ }^{32}$ de cinco pontos ( 1 a 5), sendo que para cada afirmativa positiva diminui seu valor por um (pontuação [-] 1) e para cada afirmativa negativa, cinco menos o valor (5 [-] pontuação). A pontuação final máxima de 43 (quarenta e três) pontos (pmáxima) equivale a $100 \%$ de satisfação do usuário. As pontuações para o cálculo da satisfação podem ser visualizadas na tabela 3. O resultado final é apresentado em porcentagem, através de uma fórmula inserida no software

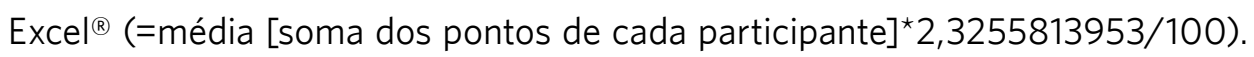
Que calcula a média dos pontos em porcentagem (Média ${ }_{\text {porcentagem }}=$ Média $_{\text {pontos }}{ }^{\star}$ 100/ máxima $/ 100$ ).

Tabela 3 - Quadro de pontuação para o cálculo da satisfação

\begin{tabular}{|c|c|c|c|c|c|c|c|c|}
\hline \multicolumn{4}{|c|}{ PARTE 01} & \multicolumn{5}{|c|}{ PARTE 02} \\
\hline \multicolumn{2}{|c|}{ Questão 01} & \multicolumn{2}{|c|}{$\begin{array}{l}\text { Questões } \\
\text { 03-04 }\end{array}$} & \multicolumn{5}{|c|}{ SUS - Afirmativas 01-10 } \\
\hline 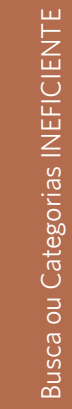 & 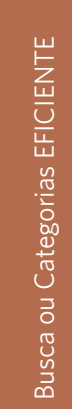 & i & $\sum$ & 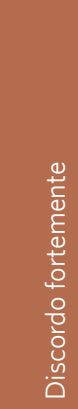 & 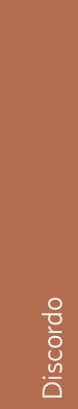 & $\begin{array}{l}\stackrel{0}{ \pm} \\
\frac{\vec{J}}{2} \\
z\end{array}$ & $\begin{array}{l}0 \\
\frac{0}{0} \\
\text { U } \\
\text { ல }\end{array}$ & 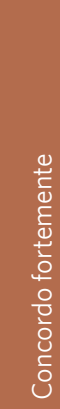 \\
\hline 0 & 1 & 0 & 1 & 1 & 2 & 3 & 4 & 5 \\
\hline \multicolumn{4}{|c|}{ Pontuação SUS - Afir. (+) } & 0 & 1 & 2 & 3 & 4 \\
\hline \multicolumn{4}{|c|}{ Pontuação SUS - Afir. (-) } & 4 & 3 & 2 & 1 & 0 \\
\hline
\end{tabular}

Fonte: Do autor.

\section{RESULTADOS E DISCUSSÕES}

A usabilidade foi analisada segundo a porcentagem de eficácia, eficiência e satisfação (ASSOCIAÇÃO BRASILEIRA DE NORMAS TÉCNICAS, 2002).

\section{Eficácia}

A taxa da eficácia foi de 73,33\% (d.p. 2,50) para o website Compra Fácil e de $76,67 \%$ (d.p. 1,51) para o website Ponto Frio (Tabela 4). A pontuação foi calculada através da média da porcentagem de subtarefas concluídas 
corretamente, conforme apresentado na Tabela 1.

Tabela 4 - Apresentação dos resultados do cálculo da eficácia

\begin{tabular}{|c|c|c|c|c|c|c|c|c|c|c|}
\hline 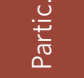 & $\mathrm{Pa} 1$ & $\mathrm{~Pa} 2$ & $\mathrm{~Pa} 3$ & $\mathrm{~Pa} 4$ & $\mathrm{~Pa} 5$ & $\mathrm{~Pa} 1$ & $\mathrm{~Pa} 2$ & $\mathrm{~Pa} 3$ & $\mathrm{~Pa} 4$ & $\mathrm{~Pa} 5$ \\
\hline \multirow{3}{*}{ 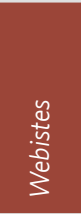 } & \multicolumn{5}{|c|}{ Compra Fácil } & \multicolumn{5}{|c|}{ Ponto Frio } \\
\hline & \multicolumn{5}{|c|}{ Subtarefas } & \multicolumn{5}{|c|}{ Subtarefas } \\
\hline & \multicolumn{5}{|c|}{ SELEÇÃO } & \multicolumn{5}{|c|}{ SELEÇÃO } \\
\hline $\begin{array}{l}\overline{0} \\
\overline{0} \\
\frac{0}{2}\end{array}$ & 1 & 1 & 0 & 0 & 0 & 1 & 1 & 1 & 1 & 1 \\
\hline 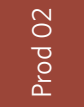 & 1 & 1 & 1 & 1 & 0 & 1 & 1 & 0 & 1 & 0 \\
\hline \multirow{2}{*}{$\begin{array}{l}m \\
0 \\
0 \\
\frac{0}{2} \\
\end{array}$} & 1 & 1 & 1 & 1 & 0 & 1 & 1 & 1 & 0 & 1 \\
\hline & \multicolumn{5}{|c|}{ CADASTRO } & \multicolumn{5}{|c|}{ CADASTRO } \\
\hline \multirow{2}{*}{ 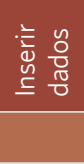 } & 1 & 1 & 1 & 1 & 0 & 1 & 1 & 1 & 1 & 1 \\
\hline & \multicolumn{5}{|c|}{ PAGAMENTO } & \multicolumn{5}{|c|}{ PAGAMENTO } \\
\hline \multirow[t]{2}{*}{$\frac{0}{\frac{0}{0}}$} & 1 & 1 & 1 & 1 & 0 & 1 & 1 & 0 & 1 & 0 \\
\hline & \multicolumn{5}{|c|}{ CONFIRMAÇÃO } & \multicolumn{5}{|c|}{ CONFIRMAÇÃO } \\
\hline 일 $\frac{\stackrel{0}{0}}{\overline{0}}$ & 1 & 1 & 1 & 1 & 0 & 1 & 1 & 0 & 1 & 0 \\
\hline 焉 & 6 & 6 & 5 & 5 & 0 & 6 & 6 & 3 & 5 & 3 \\
\hline$\partial^{\circ}$ & 100 & 100 & 83,3 & 83,3 & 0 & 100 & 100 & 50 & 83,3 & 50 \\
\hline \multirow[b]{3}{*}{ 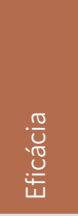 } & \multicolumn{3}{|c|}{ Média } & \multicolumn{2}{|c|}{$73,33 \%$} & \multicolumn{3}{|c|}{ Média } & \multicolumn{2}{|c|}{$76,67 \%$} \\
\hline & \multicolumn{3}{|c|}{ Desvio Padrão } & 2,5 & & \multicolumn{3}{|c|}{ Desvio Padrão } & \multicolumn{2}{|l|}{1,51} \\
\hline & \multicolumn{3}{|c|}{$\begin{array}{l}\text { Intervalo de Confiança } \\
95 \% \\
z\end{array}$} & \multicolumn{2}{|l|}{2,2} & \multicolumn{3}{|c|}{$\begin{array}{l}\text { Intervalo de Confiança } \\
95 \%\end{array}$} & \multicolumn{2}{|l|}{1,32} \\
\hline
\end{tabular}

Fonte: Do autor.

O resultado para ambos os websites está dentro da margem aceitável (7079\%), segundo Bangor, Kortum e Miller (2009). Apenas um participante (Pa5) da pesquisa não conseguiu realizar nenhuma das subtarefas no website Compra Fácil, esse mesmo participante teve dificuldades em realizar todas as subtarefas no website Ponto Frio. O resultado mostra que a subtarefa que menos pontuou foi a de escolha dos produtos, seguida de escolha da forma de pagamento e visualização da confirmação da compra, quando gera o número de pedido.

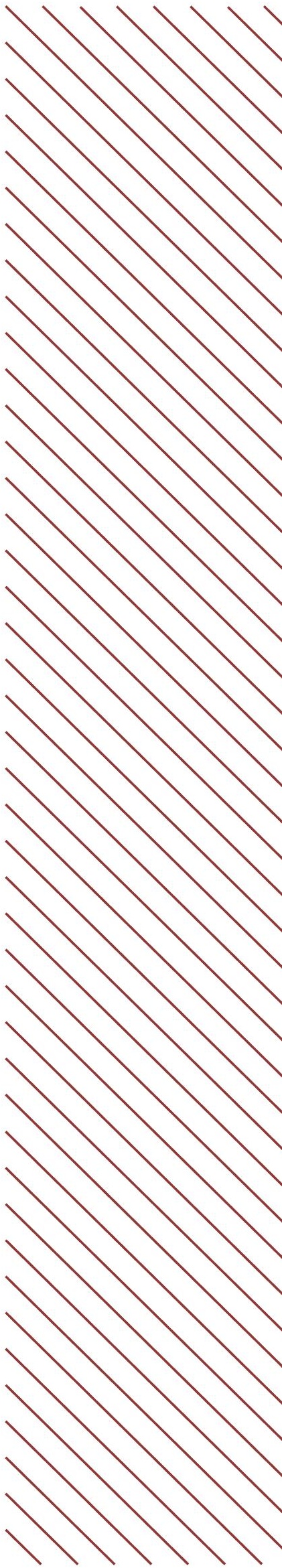




\section{Eficiência}

A taxa da eficiência foi de $51,25 \%$ (d.p. 16,27) para o website Compra Fácil e de $46,67 \%$ (d.p. 17,05) para o website Ponto Frio (Tabela 5). A pontuação foi calculada através da média da porcentagem de subtarefas concluídas corretamente dentro de certas variáveis, conforme pontuação apresentada na Tabela 2. Ambos os resultados estão dentro de uma margem considerada preocupante (abaixo de 70\%), segundo Bangor, Kortum e Miller (2009).

Tabela 5 - Apresentação dos resultados do cálculo da eficiência

\begin{tabular}{|c|c|c|c|c|c|c|c|c|c|c|}
\hline 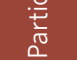 & $\mathrm{Pa} 1$ & $\mathrm{~Pa} 2$ & $\mathrm{~Pa} 3$ & $\mathrm{~Pa} 4$ & $\mathrm{~Pa} 5$ & $\mathrm{~Pa} 1$ & $\mathrm{~Pa} 2$ & $\mathrm{~Pa} 3$ & $\mathrm{~Pa} 4$ & $\mathrm{~Pa} 5$ \\
\hline \multirow{3}{*}{ 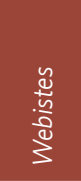 } & \multicolumn{5}{|c|}{ Compra Fácil } & \multicolumn{5}{|c|}{ Ponto Frio } \\
\hline & \multicolumn{5}{|c|}{ Subtarefas } & \multicolumn{5}{|c|}{ Subtarefas } \\
\hline & \multicolumn{5}{|c|}{ SELEÇÃO } & \multicolumn{5}{|c|}{ SELEÇÃO } \\
\hline$\frac{0}{0}$ & 5 & 5 & 0 & 0 & 0 & 5 & 5 & 1 & 6 & 1 \\
\hline 뭉 & 5 & 5 & 1 & 6 & 0 & 5 & 5 & 0 & 6 & 0 \\
\hline \multirow[t]{2}{*}{ 믈 } & 5 & 5 & 1 & 6 & 0 & 5 & 5 & 1 & 0 & 1 \\
\hline & \multicolumn{5}{|c|}{ CADASTRO } & \multicolumn{5}{|c|}{ CADASTRO } \\
\hline \multirow[t]{2}{*}{ 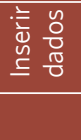 } & 7 & 6 & 2 & 8 & 0 & 7 & 3 & 2 & 8 & 2 \\
\hline & \multicolumn{5}{|c|}{ PAGAMENTO } & \multicolumn{5}{|c|}{ PAGAMENTO } \\
\hline \multirow[t]{2}{*}{$\begin{array}{l}\frac{0}{0} \\
\frac{0}{0} \\
\stackrel{0}{0}\end{array}$} & 8 & 4 & 4 & 8 & 0 & 8 & 4 & 0 & 8 & 0 \\
\hline & \multicolumn{5}{|c|}{ CONFIRMAÇÃO } & \multicolumn{5}{|c|}{ CONFIRMAÇÃO } \\
\hline $\begin{array}{ll}\frac{0}{0} \\
\text { 음 } \\
\text { 을 }\end{array}$ & 8 & 8 & 8 & 8 & 0 & 8 & 8 & 0 & 8 & 0 \\
\hline$\stackrel{\Xi}{\stackrel{0}{0}}$ & 38 & 33 & 16 & 36 & 0 & 38 & 30 & 4 & 36 & 4 \\
\hline$\delta^{\circ}$ & 79,17 & 68,75 & 33,33 & 75 & 0 & 79,17 & 62,5 & 8,33 & 75 & 8,33 \\
\hline \multirow{3}{*}{ 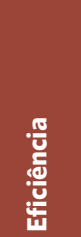 } & \multicolumn{3}{|c|}{ Média } & \multicolumn{2}{|c|}{$51,25 \%$} & \multicolumn{3}{|c|}{ Média } & \multicolumn{2}{|c|}{$46,67 \%$} \\
\hline & \multicolumn{3}{|c|}{ desvio padrão } & \multicolumn{2}{|c|}{16,27} & \multicolumn{3}{|c|}{ desvio padrão } & \multicolumn{2}{|c|}{17,05} \\
\hline & \multicolumn{3}{|c|}{$\begin{array}{l}\text { Intervalo de } \\
\text { confiança - 95\% }\end{array}$} & \multicolumn{2}{|c|}{14,26} & \multicolumn{3}{|c|}{$\begin{array}{l}\text { Intervalo de confiança } \\
-95 \%\end{array}$} & \multicolumn{2}{|c|}{14,94} \\
\hline
\end{tabular}

É possível perceber que as pontuações não diferem muito entre um website e outro na opinião dos participantes, exceto no caso Pa3 em que as pontuações diferiram entre o Compra Fácil e o Ponto Frio. Mais uma vez seguindo a tendência verificada nos resultados da eficácia, nos resultados da eficiência a seleção/escolha de produtos recebeu menor pontuação em relação à forma de pagamento e visualização do pedido. 


\section{Satisfação}

A taxa de satisfação foi de $48,84 \%$ (d.p. 6,96) para o website Compra Fácil e de $65,12 \%$ (d.p. 10,32) para o website Ponto Frio (Tabela 6). A pontuação foi calculada através da média da porcentagem das pontuações para cada questão, conforme pontuação apresentada na Tabela 3, exceto questão 02 que não pontuou por buscar apenas saber se o usuário já conhecia ou não os websites pesquisados. Ambos os resultados estão dentro de uma margem considerada preocupante (abaixo de 70\%), segundo Bangor, Kortum e Miller (2009).

\begin{tabular}{|c|c|c|c|c|c|c|c|c|c|c|}
\hline 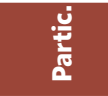 & $\mathrm{Pa} 1$ & $\mathrm{~Pa} 2$ & $\mathrm{~Pa} 3$ & $\mathrm{~Pa} 4$ & Pa5 & Pa1 & $\mathrm{Pa} 2$ & Pa3 & $\mathrm{Pa} 4$ & $\mathrm{~Pa} 5$ \\
\hline \multirow{2}{*}{ Qts } & \multicolumn{5}{|c|}{ Compra Fácil } & \multicolumn{5}{|c|}{ Ponto Frio } \\
\hline & \multicolumn{5}{|c|}{19 parte } & \multicolumn{5}{|c|}{19 parte } \\
\hline 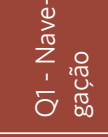 & 0 & 1 & 0 & 1 & 0 & 1 & 1 & 1 & 1 & 1 \\
\hline 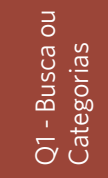 & B & B & C & B & B & B & B & C & B & C \\
\hline 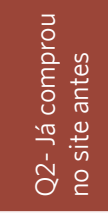 & não & não & não & não & sim & não & não & não & $\operatorname{sim}$ & não \\
\hline 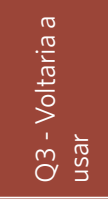 & 0 & 0 & 1 & 0 & 0 & 1 & 1 & 1 & 0 & 0 \\
\hline 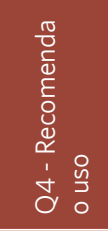 & 0 & 0 & 1 & 0 & 0 & 1 & 1 & 1 & 0 & 0 \\
\hline Qts & \multicolumn{5}{|c|}{$2^{\mathrm{a}}$ parte - SUS } & \multicolumn{5}{|c|}{$2^{\mathrm{a}}$ parte - SUS } \\
\hline 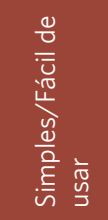 & 1 & 1 & 2 & 3 & 1 & 4 & 3 & 2 & 2 & 1 \\
\hline
\end{tabular}




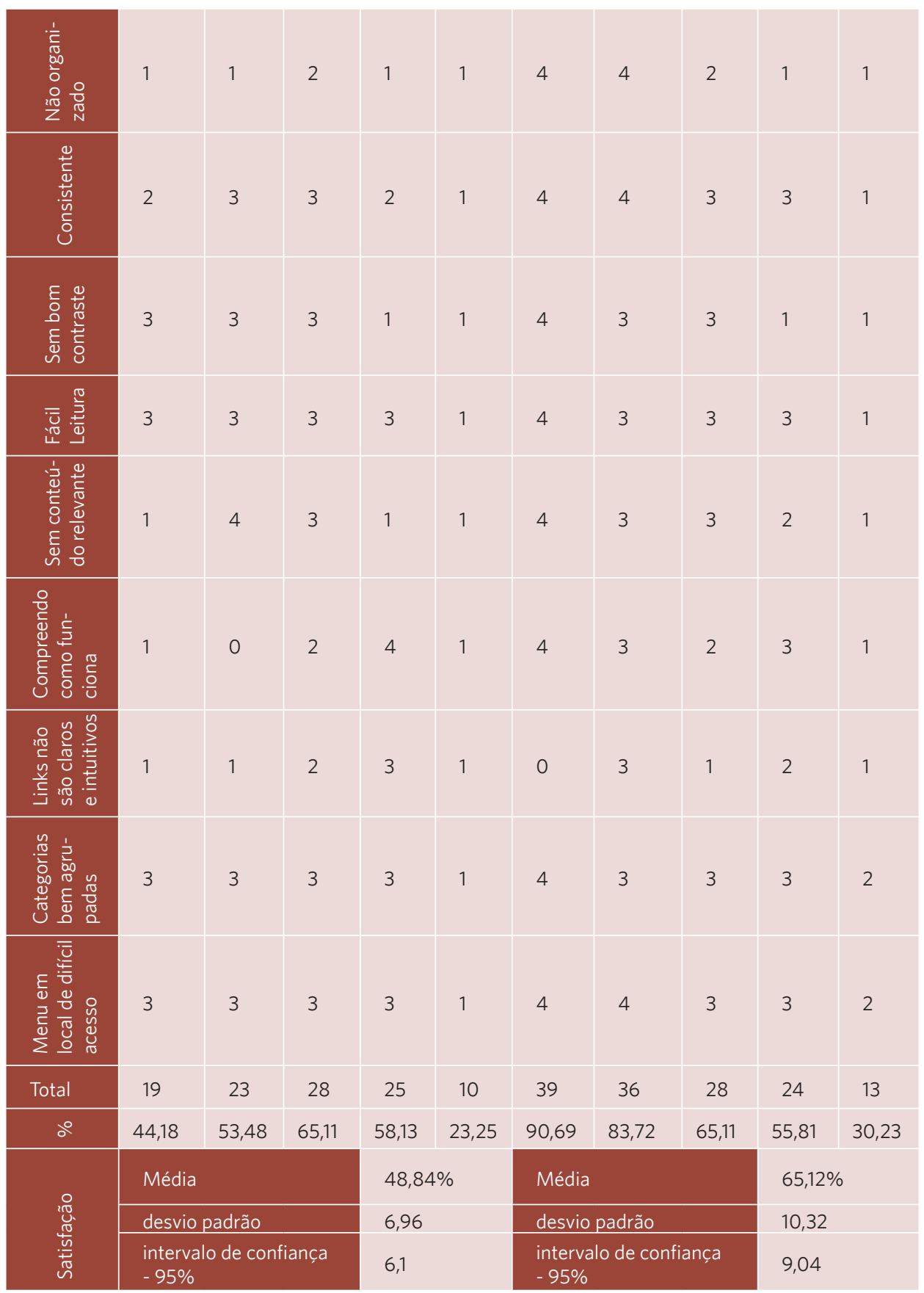

Fonte: Do autor.

Apenas um participante navegou pelas categorias e achou ineficiente para achar os produtos no website Compra Fácil e dois participantes navegaram por categorias e acharam eficiente no website Ponto Frio. Todos que navegaram pela busca no website Ponto Frio o acharam eficiente, enquanto que dois participantes acharam o sistema de busca do website Compra Fácil ineficiente. Dois participantes já conheciam os websites pesquisados, sendo que um já conhecia o Compra Fácil e outro o Ponto Frio. Três participantes voltariam a usar e recomendaria o website do Ponto Frio e apenas um participante informou que voltaria a usar o website do Compra Fácil e o recomendaria. Com relação ao 
website Ponto Frio dois participantes apresentam-se com um nível de satisfação considerado "bom" (80-90\%) por Bangor et al. (2009). Enquanto que esse nível não foi atingido por nenhum participante com relação ao website Compra Fácil. Com relação à pesquisa utilizando o SUS o website da Compra Fácil recebeu a pontuação máxima (4) uma única vez, enquanto que o website Ponto Frio recebeu essa mesma pontuação doze vezes.

\section{Apresentação do gráfico}

A figura 1 apresenta em forma de gráfico os resultados da avaliação empírica da usabilidade para ambos os websites pesquisados: Compra Fácil e Ponto Frio.

Figura 1 - Gráfico do resultado final para ambos os websites

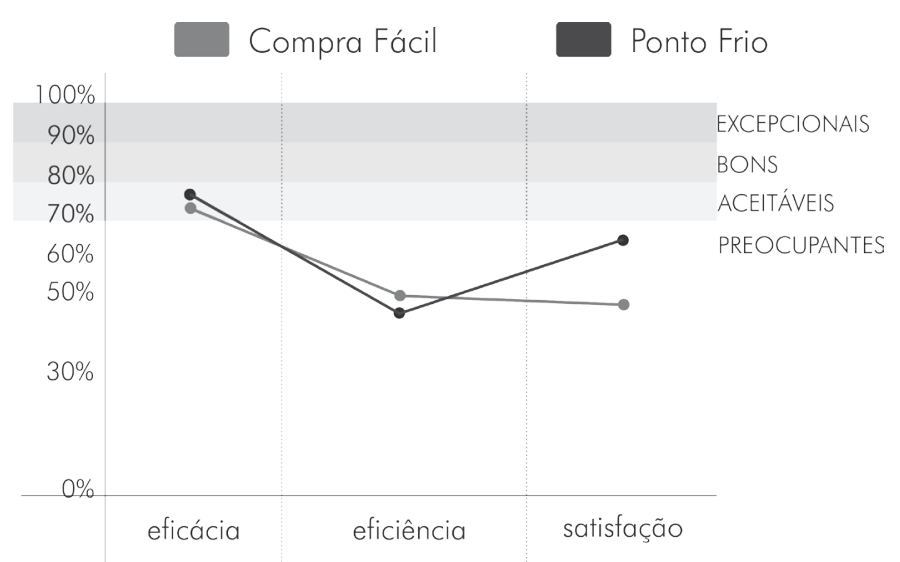

Fonte: Do autor.

É possível perceber que ambos os websites ficaram dentro da margem considerada preocupante (BANGOR; KORTUM; MILLER, 2009) tanto no resultado de eficiência quanto no resultado de satisfação do uso. $E$ apenas no resultado de eficácia é que ambos se encontram dentro da margem considerada aceitável [25]. É possível notar também que com relação aos resultados da satisfação o website Ponto Frio se posicionou em uma marca bem acima do website Compra Fácil, único momento em que os resultados dos websites tiveram uma diferença maior que cinco pontos (12 pontos de diferença).

\section{CONCLUSÃO}

Os usuários acima de 52 anos, considerados "older users" pela literatura (CZAJA, 2005; LUNN; HARPER, 2011; NAUMANEN; TUKIAINEN, 2007; ZAJICEK, 2004), estão cada vez mais inseridos no ambiente interativo das interfaces tecnológicas. Os websites de compra online são um desses ambientes que se mostra não preparado para esses usuários. Embora, em alguns casos, o 
medo e a insegurança não permitam que esses usuários (older users) se sintam confiantes em usar esses tipos de websites, muitas vezes, está relacionado à dificuldade de compreensão da interface, tanto que, nesta pesquisa, apenas metade dos participantes recomendariam um dos websites e esse número cai em relação ao outro website. As taxas de satisfação dos usuários com o website Compra Fácil não passam de 65\%, enquanto que para o Ponto Frio chegam a atingir $90 \%$ de satisfação.

Se, ao entrar em um website, o usuário tiver dificuldade em encontrar uma informação, ele irá concluir que o website é difícil de usar e se sentirá frustrado, podendo até, não retornar mais a este website. O Design de Informação tem um papel fundamental na interação e, consequentemente, na experiência do usuário, pois através do projeto de estruturação e organização da informação, dando ênfase aquelas que têm um maior peso e prioridade, o usuário consegue usar a interface com uma maior facilidade e entendimento dos recursos e características próprias dela.

Dois grandes problemas foram encontrados na realização desta pesquisa: o primeiro se tratou da seleção dos participantes que muitas vezes demonstravam receio em participar de uma interação com websites de comércio eletrônico, isso pode ser percebido no número de protocolos não respondidos ou finalizados, 15 protocolos no total, quantidade essa que ultrapassa os protocolos finalizados. O segundo problema tratou das mudanças que poderiam ocorrer nas interfaces dos dois websites, Compra Fácil e Ponto Frio, durante a realização da pesquisa.

Ainda há necessidade de aprofundar estudos da experiência do usuário no Design e criar parâmetros para o desenvolvimento de websites que se preocupem em projetar interfaces voltadas aos usuários acima de 50 anos. E ainda, que utilizem a avaliação da experiência do usuário como parte do desenvolvimento do projeto.

Estudos futuros podem abordar um número maior de participantes, em uma avaliação que possa coletar outros dados como uma avaliação mais detalhadas das expectativas e impressões iniciais (antes do uso) e ainda caminhos percorridos durante a interação, tempo gasto improdutivamente, áreas críticas e maiores inseguranças.

\section{AGRADECIMENTOS}

Este estudo foi desenvolvido com o apoio da CAPES - Coordenação de Aperfeiçoamento de Pessoal de Nível Superior. 


\section{REFERÊNCIAS}

ALBERT, B.; TULLIS, T.; TEDESCO, D. Beyond the usability lab: conducting largescale online user experience studies. São Francisco: Morgan Kaufmann, 2013.

\section{ASSOCIAÇÃO BRASILEIRA DE ERGONOMIA - ABERGO. Norma ERG BR} 1002: código de deontologia do ergonomista certificado. Ouro Preto, 2003. Disponível em: <http://www.abergo.org.br/arquivos/normas_ergbr/norma_ erg_br_1002_deontologia.pdf>. Acesso em: 20 jun. 2015.

\section{ASSOCIAÇÃO BRASILEIRA DE NORMAS TÉCNICAS - ABNT. NBR 9241-11:} Requisitos ergonômicos para trabalhos em escritórios com computadores. Parte 11 - Orientações sobre usabilidade. Rio de Janeiro, 2002.

BANGOR, A.; KORTUM, P. T.; MILLER, J. T. Determining what individual SUS scores mean: Adding an adjective rating scale. Journal Usability Studies, v. 4, n. 3, p. 114-123, 2009.

BRASIL. Conselho Nacional De Saúde. Resolução no 196, de 10 de outubro de 1996. Disponível em: <http://conselho.saude.gov.br/docs/Resolucoes/ Reso196.doc>. Acesso em: 20 jun. 2015.

CZAJA, S. J. The impact of aging on access to technology. ACM SIGACCESS Accessibility and Computing, New York, n. 83, p. 7-11, 2005.

FERNANDES, F. R.; PASCHOARELLI, L. C. A method of evaluating online usability research: a case study of the Itaú Bank Site. In: CONFERENCE PROCEEDINGS OF IADIS INTERFACES AND HUMAN COMPUTER INTERACTION, 2013, Prague. Proceedings... Prague: IADIS Press, 2013. v. 1, p. 235-239.

FERNANDES, Fabiane Rodrigues. O uso de dois websites de compras online por usuários acima de 50 anos (older users): estudo de caso. 2013. $144 \mathrm{f}$. Dissertação (Mestrado em Design) - Programa de Pós Graduação em Design, Universidade Estadual Paulista "Júlio de Mesquita Filho", Bauru, 2013.

GUASTI, Pedro. E-velhice uma nova realidade virtual. 2011. Disponível em: <http://ebit1.tempsite.ws>. Acesso em: 9 mar. 2015.

HAWTHORN, D. Designing effective interfaces for older users. 2006. 1069 p. Thesis (Doctor of Phylosophy in Computer Science) - The University of Waikato, New Zealand, 2006.

IBGE. Perfil dos idosos responsáveis pelos domicílios. 2002. Disponível em: 
<http://www.ibge.gov.br/home/presidencia/noticias/25072002pidoso. shtm>. Acesso em: 20 jun. 2015.

IBGE. Sala de imprensa: projeção da população no Brasil. 2004. Disponível em: <http://www.ibge.gov.br/home/presidencia/noticias/noticia_visualiza.php?id_ noticia=207>. Acesso em: 20 jun. 2015.

LUNN, D.; HARPER, S. Provinding assistance to older users of dynamics web content. Computers in Human Behavior, Netherlands, v. 27, n. 6, p. 2098-2107, 2011.

NAUMANEN, M.; TUKIAINEN, M. Guiding the elderly into the use of computers and internet - lessons taught and learnt. In: INTERNATIONAL CONFERENCE ON COGNITION AND EXPLORATORY LEARNING IN DIGITAL AGE, 2007. Proceedings... Algarve: CELDA 2007. p. 19-27.

NIELSEN, J.; LANDAUER, T. K. A mathematical model of the finding of usability problems. In: ACM/IFIP INTERCHI CONFERENCE, 93., 1993, Amsterdam. Proceedings... Amsterdam: ACM/IFIP INTERCHI, 1993. p. 206-213.

NIELSEN, J.; MOLICH, R. Heuristic evaluation of user interfaces. In: ACM CONFERENCE, 90., 1990, Seattle. Proceedings... Seattle: ACM CHI'90, 1990. p. 249-256.

PASCHOARELLI, L. C.; SILVA, J. C. P. Design ergonômico: uma revisão dos seus aspectos metodológicos. Conexão: Comunicação e Cultura, Caxias do Sul, v. 5, n. 10, p. 1-8, 2006.

PEW INTERNET. Pew internet protecto data memo. 2009. Disponível em: <http://www.pewinternet.org/ /media/Files/Reports/2009/PIP_ Generations_2009.pdf>. Acesso em: 20 jun. 2015.

PHIRIYAPOKANON, T. Is a big button interface enough for elderly users? towards user interface guidelines for elderly users. 2011. 61 p. Thesis (Master of Computer Engineer) - Mälardalen University, Sweden, 2011.

TULLIS, T.; ALBERT, B. Measuring the user experience: collecting, analyzing and presenting usability metrics. São Francisco: Morgan Kaufmann, 2008.

ZAJICEK, M. Sucessful and available: interface design exemplars for older users. Interacting with Computers, Oxford, n. 16, p. 411-430, 2004. 